\title{
EFECTO DE LOS TRATAMIENTOS TÉRMICOS SOBRE LAACTIVIDAD ANTIMICROBIANA DE TETRACICLINAS EN LECHE
}

\author{
Zorraquino, M. A. ${ }^{2} ;$ Althaus, R. L. ${ }^{3} ;$ Nagel, O. G. ${ }^{3} ;$ Roca, $^{1}{ }^{1}{ }^{\&}$ Molina, P. ${ }^{1}$
}

\section{RESUMEN}

La presencia de residuos de antibióticos en la leche no solamente produce un potencial riesgo para la salud del consumidor, sino que además, ocasiona serios problemas en los procesos fermentativos utilizados en la industria láctea. Por este motivo, el presente trabajo evalúa el efecto que producen aquellos tratamientos térmicos utilizados en la industria láctea $\left(40^{\circ} \mathrm{C}-10 \mathrm{~min}, 60^{\circ} \mathrm{C}-30 \mathrm{~min}, 83^{\circ} \mathrm{C}-10\right.$ $\min , 120^{\circ} \mathrm{C}-20 \mathrm{~min}$ y $140^{\circ} \mathrm{C}-10 \mathrm{~s}$ ) sobre la actividad antimicrobiana de muestras de leche fortificadas con tetraciclinas (clortetraciclina, doxiciclina, oxitetraciclina y tetraciclina). Para la medición de la pérdida de actividad antimicrobiana se empleó un bioensayo basado en la inhibición del crecimiento de Bacillus cereus subssp. mycoides ATCC11778. Para cada antibiótico se ensayaron tres concentraciones. Los datos se analizaron utilizando el modelo de regresión lineal múltiple. Los resultados señalan que la esterilización clásica $\left(120^{\circ} \mathrm{C}-20 \mathrm{~min}\right)$ produce pérdidas que superan el $90 \%$ para clortetraciclina, $84 \%$ para doxiciclina, $89 \%$ para oxitetraciclina y $91 \%$ para tetraciclina, mientras que el calentamiento a $140^{\circ} \mathrm{C}-10 \mathrm{~s}$ produce bajas pérdidas de actividad antimicrobiana ( $29 \%$ clortetraciclina, $39 \%$ doxiciclina, $17 \%$ oxitetraciclina y $29 \%$ tetraciclina), similares a la baja pasteurización $\left(60^{\circ} \mathrm{C}-30\right.$ minutos) ( $31 \%$ clortetraciclina, $18 \%$ doxiciclina, $23 \%$ oxitetraciclina y $21 \%$ tetraciclina). Los tratamientos térmicos de laboratorio $\left(40^{\circ} \mathrm{C}-10 \mathrm{~min}\right.$ y $\left.83^{\circ} \mathrm{C}-10 \mathrm{~min}\right)$ ocasionan pérdidas de actividad antimicrobiana aún menores.

Palabras clave: antibióticos, tetraciclinas, termoestabilidad, inactivación térmica, leche.

\section{SUMMARY}

Heat treatment effects on the antimicrobial activity of tetracycline in milk.

The presence of antibiotic residues in milk is not only a potential consumer risk, but may also cause serious problems in the fermentation processes used in the dairy industrythe aim of the study was, therefore, to analyze the effect of different heat treatments $\left(40^{\circ} \mathrm{C}-10 \mathrm{~min}, 60^{\circ} \mathrm{C}-30 \mathrm{~min}, 83^{\circ} \mathrm{C}-10\right.$ min, $120^{\circ} \mathrm{C}-20 \mathrm{~min}$ and $140^{\circ} \mathrm{C}-10 \mathrm{~s}$ ) on the antimicrobial activity of milk samples fortified with tetracycline (chlortetracycline, doxycycline, oxytetracycline and tetracycline). To measure the loss

1.- Instituto de Ciencia y Tecnología Animal. Universidad Politécnica de Valencia. Camino de Vera, 14, 46071 Valencia, España.

2.- Departamento de Producción Agraria. Universidad Pública de Navarra. Campus de Arrosadía, 31006 Pamplona, España

3.- Cátedra de Biofísica, Departamento de Ciencias Básicas, Universidad Nacional del Litoral. Kreder 2805 (3080) Esperanza, provincia de Santa Fe.

Manuscrito recibido el 10 de noviembre de 2010 y aceptado para su publicación el 10 de febrero de 2011. 
of antimicrobial activity a bioassay based on inhibition of growth of de Bacillus cereus subssp. mycoides ATCC11778 was used. For each antibiotic three concentrations were testedThe data were analyzed using a multiple linear regression model. The results showed that classic sterilization $\left(120^{\circ} \mathrm{C}-20 \mathrm{~min}\right)$ produced losses that exceeded $90 \%$ for chlortetracycline, $84 \%$ doxycycline, $89 \%$ oxytetracycline and $91 \%$ tetracycline, while the heat treatment $140^{\circ} \mathrm{C}-10$ s produced little loss in antimicrobial activity (29\% chlortetracycline, $39 \%$ doxycycline, $17 \%$ oxytetracycline and $29 \%$ tetracycline), similar to low temperature pasteurization $\left(60^{\circ} \mathrm{C}-30 \mathrm{~min}\right)(31 \%$ chlortetracycline, $18 \%$ doxycycline, $23 \%$ oxytetracycline and $21 \%$ tetracycline). Laboratory heat treatments $\left(40{ }^{\circ} \mathrm{C}-10 \mathrm{~min}\right.$ and $83{ }^{\circ} \mathrm{C}-10 \mathrm{~min}$ ) caused little loss of antimicrobial activity

Key words: antibiotics, tetracycline, thermostability, heat inactivation, milk. 